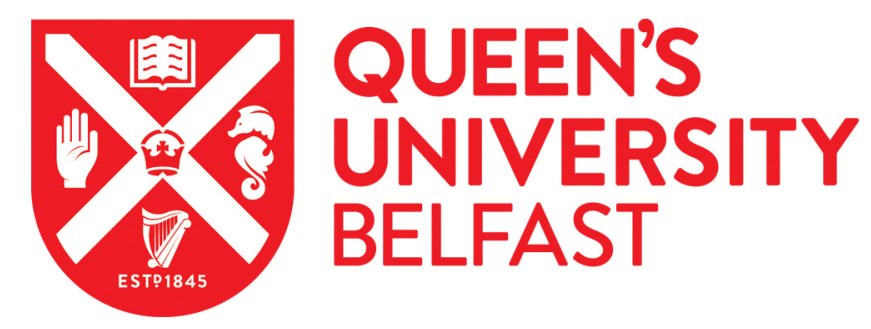

\title{
Analysis of Latent Tracks for MeV protons in CR-39
}

Kar, S., Borghesi, M., Romagnani, L., Takahashi, S., Zayats, A., Malka, V., Fritzler, S., \& Schiavi, A. (2007). Analysis of Latent Tracks for MeV protons in CR-39. Journal of Applied Physics, 101(4), [044510]. https://doi.org/10.1063/1.2433744

\section{Published in:}

Journal of Applied Physics

\section{Document Version:}

Publisher's PDF, also known as Version of record

\section{Queen's University Belfast - Research Portal:}

Link to publication record in Queen's University Belfast Research Portal

\section{Publisher rights}

(C) 2007 American Institute of Physics

\section{General rights}

Copyright for the publications made accessible via the Queen's University Belfast Research Portal is retained by the author(s) and / or other copyright owners and it is a condition of accessing these publications that users recognise and abide by the legal requirements associated with these rights.

Take down policy

The Research Portal is Queen's institutional repository that provides access to Queen's research output. Every effort has been made to ensure that content in the Research Portal does not infringe any person's rights, or applicable UK laws. If you discover content in the Research Portal that you believe breaches copyright or violates any law, please contact openaccess@qub.ac.uk. 


\section{AIP Appilied Physics}

\section{Analysis of latent tracks for MeV protons in CR-39}

S. Kar, M. Borghesi, L. Romagnani, S. Takahashi, A. Zayats et al.

Citation: J. Appl. Phys. 101, 044510 (2007); doi: 10.1063/1.2433744

View online: http://dx.doi.org/10.1063/1.2433744

View Table of Contents: http://jap.aip.org/resource/1/JAPIAU/v101/i4

Published by the American Institute of Physics.

\section{Related Articles}

Position and energy-resolved particle detection using phonon-mediated microwave kinetic inductance detectors Appl. Phys. Lett. 100, 232601 (2012)

High-resolution Thomson parabola for ion analysis

Rev. Sci. Instrum. 82, 113504 (2011)

The response of CR-39 nuclear track detector to 1-9 MeV protons

Rev. Sci. Instrum. 82, 103303 (2011)

Increasing the energy dynamic range of solid-state nuclear track detectors using multiple surfaces Rev. Sci. Instrum. 82, 083301 (2011)

A high-sensitivity angle and energy dipersive multichannel electron momentum spectrometer with $2 \pi$ angle range

Rev. Sci. Instrum. 82, 033110 (2011)

\section{Additional information on J. Appl. Phys.}

Journal Homepage: http://jap.aip.org/

Journal Information: http://jap.aip.org/about/about_the_journal

Top downloads: http://jap.aip.org/features/most_downloaded

Information for Authors: http://jap.aip.org/authors

\section{ADVERTISEMENT}

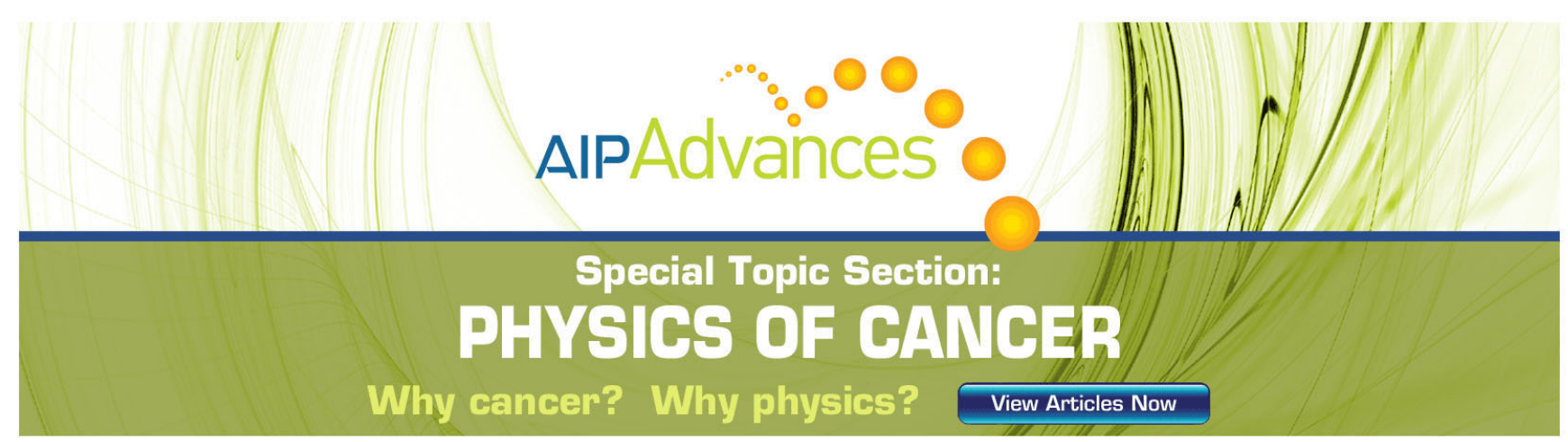




\title{
Analysis of latent tracks for MeV protons in CR-39
}

\author{
S. Kar, ${ }^{\text {a) }}$ M. Borghesi, L. Romagnani, S. Takahashi, and A. Zayats \\ Department of Physics and Astronomy, Queen's University of Belfast, Northern Ireland BT7 1NN, \\ United Kingdom \\ V. Malka and S. Fritzler \\ Laboratoire d'Optique Appliquee, ENSTA, 91128 Palaiseau, France
}

\author{
A. Schiavi \\ Dipartimento di Energetica, Universita di Roma, "La Sapienza”, 00185 Roma, Italy
}

(Received 4 September 2006; accepted 28 November 2006; published online 26 February 2007)

\begin{abstract}
For protons of energy up to a few MeV, the temporal evolution of etched latent tracks in CR-39 nuclear track detector has been numerically modeled by assuming that the electronic energy loss of the protons governs the latent track formation. The technique is applied in order to obtain the energy spectrum of high intensity laser driven proton beams, with high accuracy. The precise measurement of the track length and areal track density have been achieved by scanning short etched, highly populated CR-39 employing atomic force microscope. (C) 2007 American Institute of Physics.
\end{abstract}

[DOI: $10.1063 / 1.2433744]$

\section{INTRODUCTION}

"California Resin No. 39" (CR-39) track detector is commonly used for particle detection in various fields of science such as astrophysics, nuclear physics, life science, and geophysics. ${ }^{1}$ The main advantage of CR-39 as a detector in these application areas is its insensitivity to electromagnetic radiation and lightly ionizing particles such as electrons. Incident ions (having energy above $100 \mathrm{keV} /$ nucleon) are detected by the latent tracks left in the CR-39, in the form of broken molecular chains and free radicals, revealed after etching with caustic alkali solutions such as $\mathrm{NaOH}$ or $\mathrm{KOH}$. The length and the opening diameter of the etched tracks are two observables providing information about charge, mass, and energy of the incident particle. In recent years CR-39 has been widely used as a detector for high intensity laser driven ions in order to obtain beam spatial and spectral information. $^{2-4}$ These application areas demand absolute calibration of the etched tracks against the physical properties (e.g. charge, mass and energy) of the incident particle.

Since there is no precise analytical model for particle registration in solid state particle track detectors, the absolute calibration can only be obtained experimentally. However, it is a time demanding task to perform such calibrations for a wide variety of particles of different masses, charges and energies at different etching conditions (such as temperature, molarity etc.). Therefore, an intermediate approach can be applied, such as extrapolating the available experimental calibrations and using numerical algorithms, based on realistic approximations.

Extraction of mass, charge and energy of an incident particle from the etched track diameter requires crude approximations, unlike the calibration based on track length measurement. ${ }^{5}$ In this paper a simple way of calibrating the etched track length of protons with energy up to a few MeV

\footnotetext{
a) Author to whom correspondence should be addressed; electronic mail: s.kar@qub.ac.uk
}

is discussed, which, in principle, can be extrapolated to particles with any charge, mass and energy. After comparing experimental calibration for light incident particles (such as protons) of energy up to a few MeV/nucleon with Monte Carlo simulation, it has been assumed that the electronic energy loss (EEL) (energy loss of incident particles by virtue of inelastic collisions with target electrons, either free or bound with the lattice/atoms/molecules) of the incident particle governs the amount of damage in latent tracks. We propose the nomenclature "electronic energy loss model" for the latent track formation. The remaining part of the letter is organized as follows. Following a brief introduction on etched track formation in solid state particle track detectors, a formal description of the EEL model is given. Employing the model, the response of the CR-39 for protons of different energies is obtained. As an implementation of the technique, the energy spectrum of protons, emitted from high intensity laser irradiated thin foils, is finally obtained. Precise measurements of the etched track parameters and areal track density have been achieved employing atomic force microscope (AFM).

\section{FUNDAMENTALS OF LATENT TRACKS}

The damaged plastic in a latent track dissolves more rapidly in the etcher than the undamaged matter outside the track. This is because the etching rate of the monomer increases with a decrease in its average molecular weight. ${ }^{6}$ The etch rate of the undamaged matter (bulk etch rate, $V_{B}$ ) depends mainly on the type of detector and etching conditions. On the other hand, the etch rate of the damaged monomer in a latent track (track etch rate, $V_{T}$ ) depends not only on these two factors but also depends strongly on the incident ion's energy loss properties: $d E / d x=f(Z, E)$, where $Z$ is the charge of the ion $\left(d E / d x\right.$ stands here for any type of energy loss). ${ }^{7}$ As the energy loss of the particle at some point of its trajec- 
tory is a function of its energy at that point, etching of a latent track gives a time varying track length $(L)$. Hence the track etch rate can be expressed as ${ }^{8}$

$$
V_{T}(t)=\frac{1}{\cos \theta}\left[\frac{d L(t)}{d t}+V_{B}\right]
$$

where $\theta$ is the angle of incidence of the incident particle. Alternatively, the track length is given by

$$
L(t)=\cos \theta \int_{0}^{t} V_{T}\left(t^{\prime}\right) d t^{\prime}-V_{B} t
$$

The track length is a physical observable and can be measured directly by looking at the tracks at different etching times. The ratio between the etch rates of the damaged and undamaged plastic is known as the reduced etch rate $(V)$. This parameter is of fundamental interest as it mainly depends on the particle energy loss along its trajectory inside the CR-39.

\section{EEL MODEL OF LATENT TRACK FORMATION}

The mechanism, described by the relevant $d E / d x$, by which a particle loses its energy as it slows down and comes to rest, is central to any attempt of understanding the latent track formation. The total energy loss of a projectile ion is the sum of energy transferred, via elastic or inelastic collisions, to electrons and nuclei/atoms/molecules of the target matter. These two routes of energy loss are, respectively, called electronic energy loss and nuclear energy loss. The effect of electronic stopping is either to excite the electrons to higher energy levels or to loosen or eject them from the atoms they are bound with. The latter process creates charge centers in the solid and the ejected energetic electrons (delta electrons) produce further excitation and ionization in the neighborhood. Loss via nuclear stopping can be described as the energy transferred to the target nuclei/atoms/molecules via elastic scattering. The amount of energy loss via each of the mechanisms depends on physical and chemical properties of the incident and target particles such as mass, energy and binding energy of the lattice (for the target molecule only). In the case of CR-39 exposed to protons of energy more than $100 \mathrm{keV}$ (threshold detection energy), the dominant interaction is the one between the incident particle and electrons attached to atoms within the lattice. The electronic and nuclear energy losses of incident protons, of different energies, in CR-39 are computed employing Monte Carlo simulation, SRIM, ${ }^{9}$ as shown in Fig. 1. It can be clearly seen that for $\mathrm{MeV}$ protons, the electronic energy loss is three orders of magnitude higher than the nuclear energy loss.

Among the ionization processes, the relative dominance of the primary (by the incident particle) and secondary (by the delta electrons) ionization and excitation towards the total damage in the latent track is not yet clearly known. In inorganic solids such as crystals and glasses, the case is simple because of their extreme insensitivity to electron bombardment. But in the case of polymers it is probable that both primary and secondary ionizations contribute, and consequently both these processes will need to be considered in a complete theory.

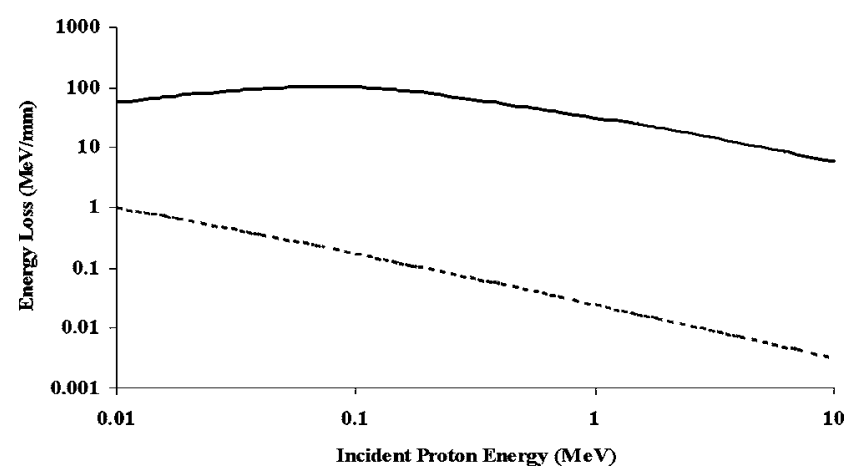

FIG. 1. Electronic (solid line) and nuclear (dotted line) energy losses of protons propagating through CR-39, as obtained from Monte Carlo simulation SRIM.

Several energy loss theories have been developed describing mechanisms of the formation of latent track by scaling the track etch rate to fit with the experimentally observed $L(t)$. The original idea was based on the total energy loss of the incident particle, which can be calculated by the BetheBloch formula. ${ }^{10}$ The recently developed restricted energy loss (REL) model ${ }^{11}$ assumes that the response of the polymers depends on the restricted energy loss (the fraction of the total energy loss that produces delta electrons of less than a given energy) of the incident particle. For incident particle energy of a few $\mathrm{MeV} /$ nucleon, the restricted energy loss is approximately equal to the total energy loss, ${ }^{6}$ which is again approximately equal to EEL (as can be inferred from Fig. 1). Therefore, we can hypothesize that for lighter particles, such as protons, EEL is mostly responsible for the breaking of molecular chains of monomer along the particle trajectory and formation of latent tracks.

EEL of proton at different detector depths can be simulated precisely (within $5 \%$ error $^{12}$ ) by employing the Monte Carlo simulation SRIM (Ref. 9) (a freeware simulation package available in the world wide web). Therefore, once the calibration for $V$ versus EEL is obtained, one can calculate $L(t)$ for a given particle energy.

\section{THE CALIBRATION}

The calibration between $V$ and EEL has been obtained by considering experimentally obtained values of $V$ versus detector depth and simulated (employing SRIM) EEL versus detector depth. Dorschel et $\mathrm{al}^{8}{ }^{8}$ have experimentally measured $L(t)$ for several different energies of protons. In this case, the CR-39 detectors were exposed normally to protons of different energies and were etched in a controlled way for different times. The lengths of the etched tracks were measured by breaking the CR-39 into several pieces perpendicular to its surface. The track etch rate was then calculated from the $L(t)$ following Eq. (1). The calculation of the bulk etch rate was done simply by measuring the thickness of the etched detector at different etching times. Figure 2 thus shows the set of points (EEL, $V$ ) obtained for different detector depths, for two proton energies, viz., 0.77 and $0.87 \mathrm{MeV}$. The linear fit of the data points is taken as an empirical relation in order to reconstruct the reduced etch rate at different detector depths for a given proton energy. 


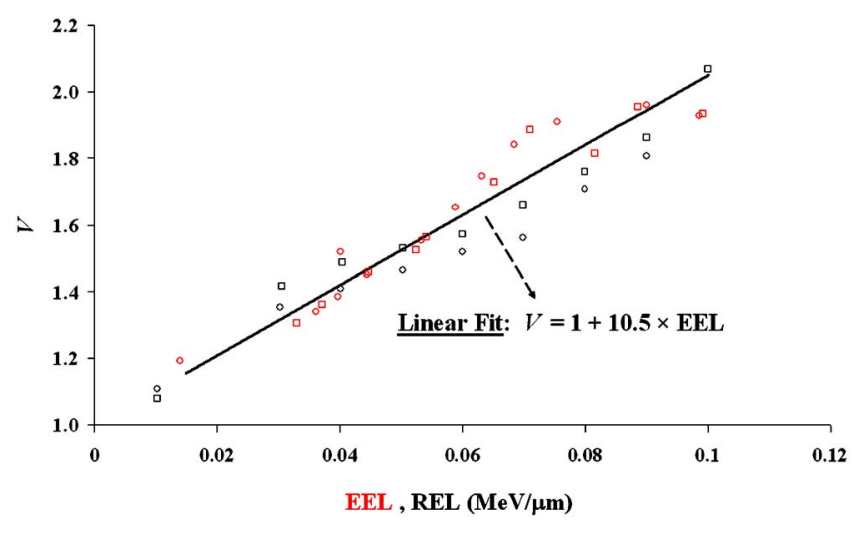

FIG. 2. (Color online) Variation of reduced etch rate with EEL (red) and REL (black) obtained for the tracks caused by protons of energies of $0.77 \mathrm{MeV}$ (circle) and $0.87 \mathrm{MeV}$ (square). The solid line is a linear fit to the data points.

Figure 2 also shows the variation of the reduced etch rate with the REL, calculated by Dorschel et al., ${ }^{5}$ in order to illustrate that in $\mathrm{MeV}$ energy range of incident protons, both REL and EEL are fairly close to each other.

We developed a computer code in order to calculate the time dependent track length using the depth dependent reduced etch rate. The algorithm used in the one-dimensional (1D) code is as follows. As the code uses the depth dependent track etch rate, the total thickness of the detector is divided into small steps $\Delta x$. Using the track etch rate at a given depth of the detector, the code calculates the time $(\Delta t)$ required for the complete etching of the thickness step, $\Delta x$. In the next step, the code calculates the thickness $\Delta x^{\prime}$ of the undamaged matter that could be etched during this etching time $\Delta t$ at the bulk etch rate. $\Delta=\Delta x-\Delta x^{\prime}$ thus gives the track length after that small interval of etching time. These steps are repeated for all the thickness steps in the detector. The simulated $L(t)$ for different proton energies are shown in Fig. 3. We verified that the graphs for protons of energies of 0.77 and $0.87 \mathrm{MeV}$ agree with the experimental measurements reported by Dorschel et al. ${ }^{8}$

For incident proton energy of $100 \mathrm{keV}$, as can be seen from Fig. 3, the latent track length saturates after a very short etching time (tens of minutes), depending on the etching conditions. For this range of etching time, the etched track length is similar for $\mathrm{MeV}$ or higher energy protons (Fig. 3) as Bragg's peak of energy deposition lies in the unetched depth of the detector. Therefore the resolution of track length measurement determines the upper limit of measurable ion energy range.

\section{PROTON ENERGY SPECTRUM}

As an application of the presented technique of track analysis, the energy spectrum of laser produced proton beam is obtained from an exposed CR-39, shown in Fig. 4(a). In the experiment, carried out at LOA laboratory, France, the proton beam was generated from a $10 \mu \mathrm{m}$ thick $\mathrm{Al}$ foil irradiated by Ti:sapphire laser delivering peak intensity of 2 $\times 10^{19} \mathrm{~W} / \mathrm{cm}^{2}$ on the target. ${ }^{4}$ Figure 4(a) shows optical scan (in transmission mode) of typical CR-39 obtained from the experiment, where the gray level corresponds to proton track density. The central white line corresponds to the shadow of a $50 \mu \mathrm{m}$ Ta wire placed in between the proton source and the CR-39. The detector was covered by $40 \mu \mathrm{m}$ thick $\mathrm{Al}$ foil in order to protect from heavy ions and debris from the target. Therefore the tracks found in CR-39 were due to the protons of energy more than $1.85 \mathrm{MeV}$ (the stopping energy of the

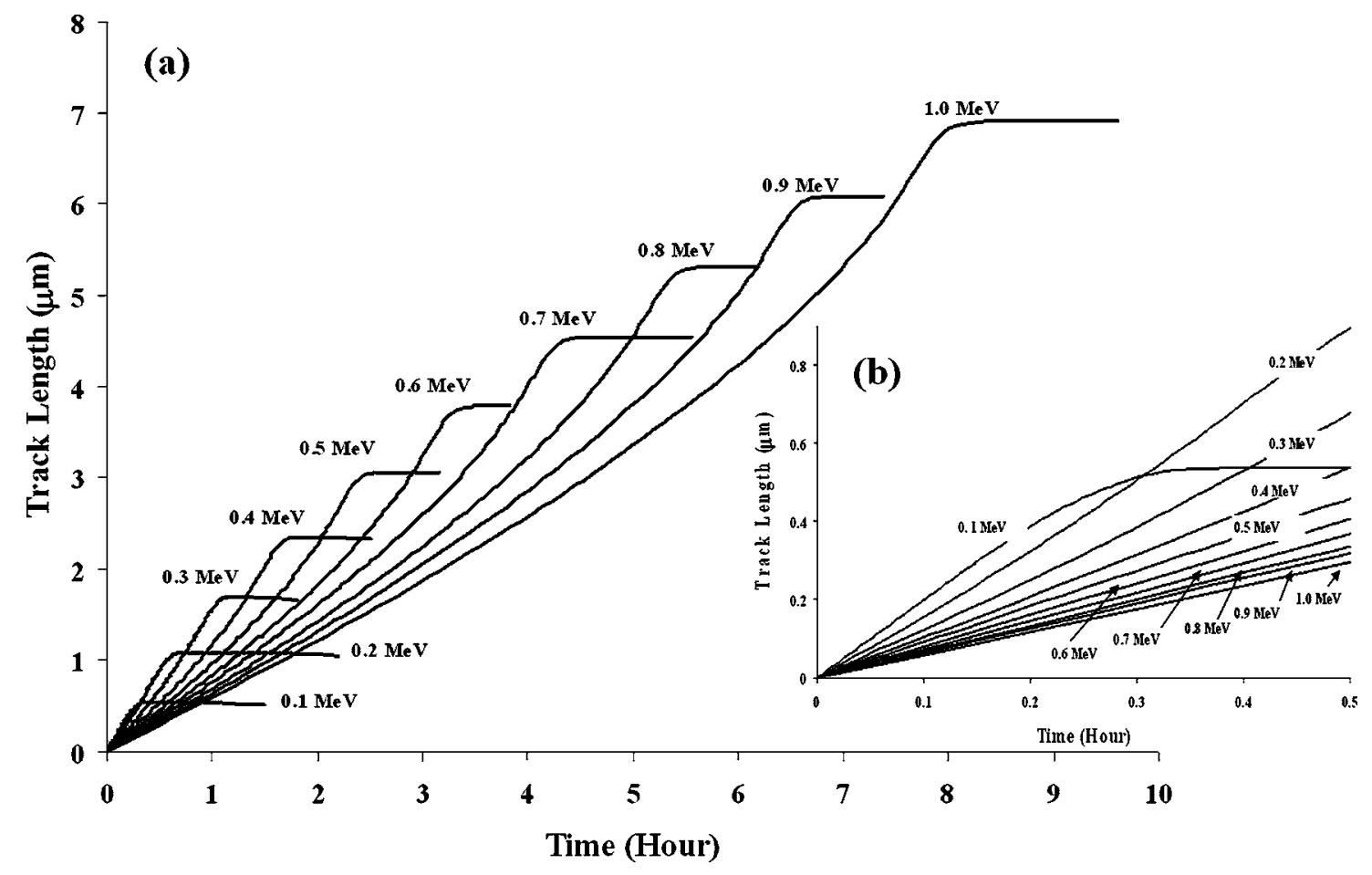

FIG. 3. (a) Simulated track lengths as a function of etching time for different incident proton energies. (b) Zoom-in view of (a) for the first half hour of etching. 

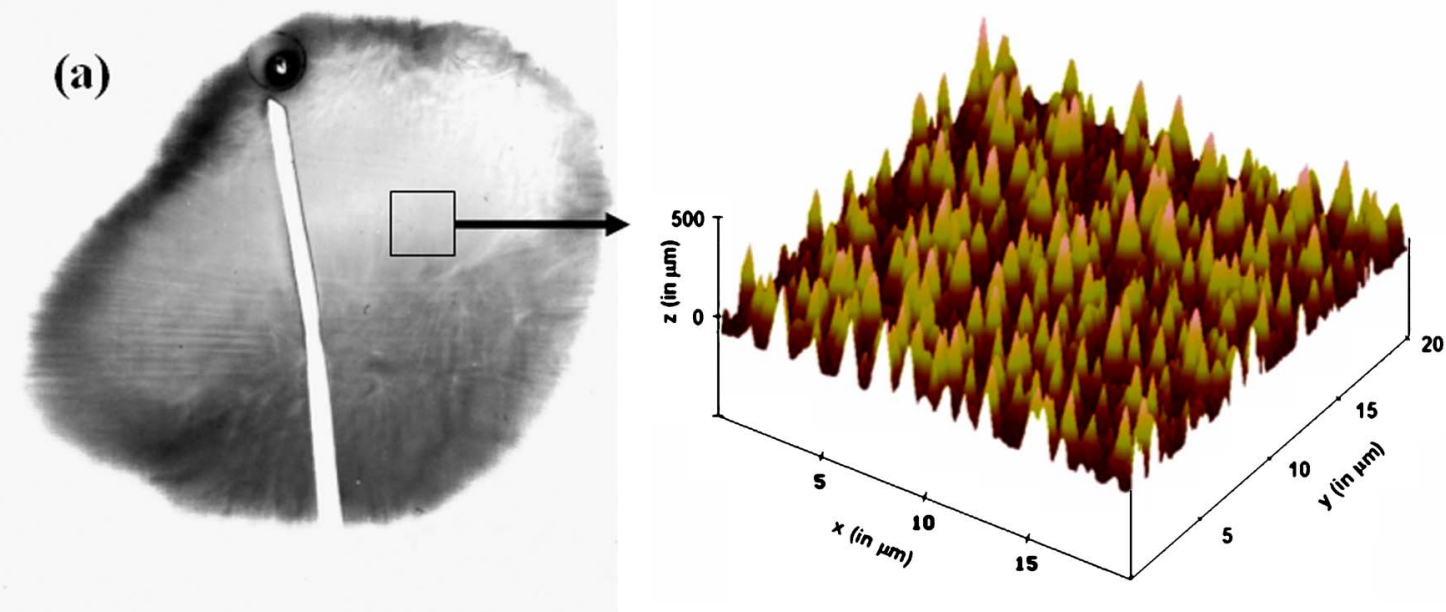

FIG. 4. (a) Optical scan (in transmission mode), using flatbed scanner, of experimentally obtained CR-39 exposed to laser driven proton beam. (b) Inverted 3D image of a $20 \times 20 \mu \mathrm{m}^{2}$ surface area [sampled from the area marked by the square in (a)] obtained by using AFM. The humps in the 3D image are the shape of the conical tracks.

$40 \mu \mathrm{m} \mathrm{Al} \mathrm{filter).} \mathrm{In} \mathrm{order} \mathrm{to} \mathrm{avoid} \mathrm{overlapping} \mathrm{of} \mathrm{tracks,}$ CR-39 was etched for only 15 min in $70 \%$ by mass of $\mathrm{NaOH}$ aqueous solution, at a temperature of $80{ }^{\circ} \mathrm{C}$. Even after short etching, we found extremely difficult analyzing the highly populated tracks in CR-39 by using an optical microscope even of very high magnification. The drawback of an optical microscope is the poor resolution in counting the tracks and resolving the overlapping. Due to the large density of tracks, it was also not practically feasible to obtain the track lengths by following the experimental method adopted by Dorschel et $a{ }^{8}{ }^{8}$ Therefore, AFM (Ref. 13) was employed in order to obtain surface topography of the etched CR-39. The AFM was operated in tapping mode in order to achieve high resolution without inducing destructive frictional forces. ${ }^{13}$ Figure 4(b) shows the AFM scan of a small part of CR-39 marked in Fig. 4(a).

A number of AFM scans of different domains within the CR-39 having uniform proton fluxes were taken. Each scan was analyzed in order to obtain a histogram of number of tracks for different track lengths, in small increments. Here we have chosen incremental bins of $20 \mathrm{~nm}$. The error in the measurement of the track length was less than a few nanometers and the error in track density was less than a few percent of the obtained value. Using the calibration shown in Fig. 3, the energy of the protons corresponding to each track length was calculated. A correction in the proton energy was introduced to take into account the Al filter used to protect the CR-39 in the experiment. The corrections were obtained by simulating (using SRIM) the energy of the transmitted protons, for a given incident proton energy, through $40 \mu \mathrm{m}$ thick Al foil. The energy spectrum of the protons thus obtained is shown in Fig. 5.

\section{CONCLUSION}

In conclusion, an EEL model of CR-39 track formation is proposed which is based on the fact that at low energy, the damage in plastic track detectors by light ions is mainly caused by their electronic energy losses. The technique offers a viable way of calibrating tracks in CR-39 against incident particle parameters, alternative to extensive experimental procedures. The use of AFM for scanning of etched CR-39 has been demonstrated to be extremely efficient in measuring etched track parameters with high spatial resolution, of the order of nanometers. Present day techniques for measuring the energy spectrum of a broadband ion beam (e.g., laser produced) are limited to provide either spatially integrated spectra with coarse energy resolution (as in the case of techniques employing nuclear activation measurements ${ }^{14}$ and deposited dose measurement in radiochromic films ${ }^{15}$ ) or high precision, high energy resolution spectra along a single line of sight to the diagnostic, such as with magnetic spectrometers. ${ }^{3}$ The technique of obtaining energy spectra from CR-39, as demonstrated in this article, can potentially be extended in order to obtain spatially resolved complete ion spectra with high energy resolution, by employing stacks of thin nuclear track detectors, multiple cycles of etching, and AFM analysis. By suitably choosing the filter thickness one can detect even lower proton energies than extracted in this paper. This is particularly useful in cases (such as for laser-accelerated ion beams) in which the ion beam spectrum has an angular dependence or varies across the beam cross section. ${ }^{16}$

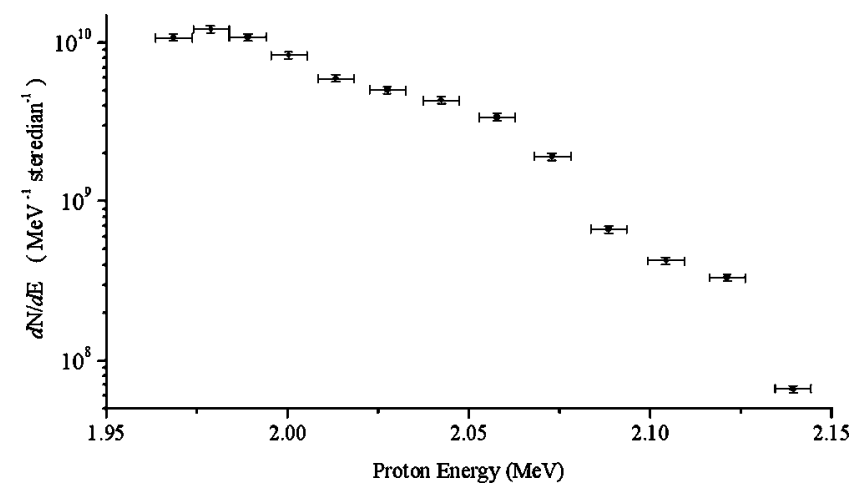

FIG. 5. Energy spectrum of the laser driven protons, collected by the CR-39 (shown in Fig. 4) in the experiment. 


\section{ACKNOWLEDGMENTS}

The authors acknowledge support for the experiment from EU Access Contract No. HPRI-1999-CT-00086 and QUB/IRCEP scheme. The authors also acknowledge useful discussions with M. Zepf (QUB, UK) and technical support from A. J. Tuner (QUB, UK).

${ }^{1}$ R. K. Singh, B. Mitra, N. Durgaprasad, S. Biswas, M. N. Vahia, J. S. Yadav, A. Dutta, and J. N. Goswami, Astrophys. J. 374, 753 (1991); K. Oschlies, R. Beaujean, and W. Enge, ibid. 45, 776 (1989); P. B. Price, M. L. Tincknell, G. Tarle, S. P. Ahlen, K. A. Frannkel, and S. Perlmutter, Phys. Rev. Lett. 50, 566 (1983); E. B. Benton, W. Heinrich, T. A. Parnell, T. W. Armstrong, J. H. Derrickson, G. J. Fishman, A. L. Frank, J. W. Watts, and B. Wiegel, Nucl. Tracks Radiat. Meas. 20, 75 (1992); S. Nakamura, S. Orito, T. Suzuki, T. Doke, H. Ichinose, K. Kuwahara, and K. Ogura, Phys. Lett. B 263, 529 (1991).

${ }^{2}$ M. Zepf et al., Phys. Plasmas 8, 2323 (2001); M. Zepf et al., Phys. Rev. Lett. 90, 064801 (2003); F. Lindau, O. Lundh, A. Persson, P. McKenna, K. Osvay, D. Batani, and C. G. Washlstrom, ibid. 95, 175002 (2005).

${ }^{3}$ B. M. Hegelich et al., Nature (London) 439, 441 (2006); L. Willingale et al., Phys. Rev. Lett. 96, 245002 (2006).

${ }^{4}$ M. Borghesi et al., Phys. Rev. Lett. 94, 195003 (2005).
${ }^{5}$ B. Dorschel, D. Hermsdorf, and K. Kadner, Radiat. Meas. 35, 183 (2002). ${ }^{6}$ R. L. Fleischer, P. B. Price, and R. M. Walker, J. Appl. Phys. 36, 3645 (1965); R. L. Fleischer, P. B. Price, and R. M. Walker, Nuclear tracks in Solids, Principles and Applications (University of California Press, Berkeley, 1975).

${ }^{7}$ W. Enge, Radiat. Meas. 25, 11 (1995)

${ }^{8}$ B. Dorschel, D. Fulle, H. Hartmann, D. Hermsdorf, K. Kadner, and C. Radlach, Radiat. Prot. Dosim. 69, 267 (1997); B. Dorschel, R. Bretschneider, D. Hermsdorf, K. Kadner, and H. Kuhne, Radiat. Meas. 31, 103 (1999).

${ }^{9}$ http://www.srim.org; J. F. Ziegler, The Stopping and Range of Ions in Matter (Pergamon, New York, 1985), Vol. 1.

${ }^{10}$ U. Fano, Annu. Rev. Nucl. Sci. 13, 1 (1963); L. C. Northcliffe, ibid. 13, 67 (1963).

${ }^{11}$ E. V. Benton and R. P. Henke, Nucl. Instrum. Methods 58, 241 (1968); E. V. Benton and W. D. Nix, ibid. 67, 343 (1969).

${ }^{12} \mathrm{http} / / /$ www.srim.org/SRIM/SRIMPICS/STOPPLOTS.htm

${ }^{13}$ R. Wiesendanger, Scanning Probe Microscopy (Springer, Heidelberg, 1998).

${ }^{14}$ R. Snavely et al., Phys. Rev. Lett. 85, 2945 (2000); P. McKenna et al., Phys. Rev. E 70, 036405 (2004).

${ }^{15}$ E. Breschi, M. Borghesi, M. Galimberti, D. Gulietti, L. A. Gizzi, L. Romagnani, A. Schiavi, and O. Willi, Laser Part. Beams 22, 393 (2004).

${ }^{16}$ M. Roth et al., Phys. Rev. ST Accel. Beams 5, 061301 (2002). 